\title{
High power Bessel-like laser beam by thin disk geometry
}

\author{
R. Aghbolaghi ${ }^{1 \star}$, S. Batebi ${ }^{1}$ and J. Sabaghzadeh ${ }^{2}$ \\ ${ }^{1}$ Department of Physics, University of Guilan, Rasht, Iran. \\ ${ }^{2}$ Iranian National Center of Laser Science and Technology (INCLST), Tehran, Iran.
}

Accepted 18 June, 2012

\begin{abstract}
We have numerically shown that a high power Bessel-Gauss beam can be generated by a solid state thin disk laser using an axicon-based resonator. Ytterbium ions doped in the Yttrium Aluminum Garnet (YAG) crystal was utilized in this configuration as an active medium. We obtained the output power, the intensity as well as phase profiles on the output coupler and the active medium.
\end{abstract}

Key words: Axicon based resonator, Thin disk laser, Bessel like beam.

\section{INTRODUCTION}

Diffraction-free beam and optical invariant field are special cases of optical wave propagation that have caught general attention in the last decade. Temporal solitons were the first class of optical signals to be recognized as being invariant under propagation (Hasegawa and Tapert, 1973) and to be experimentally demonstrated (Mollenaure et al., 1980). But, they require a nonlinearity to preserve their shape. It was pointed out by Durnin that nonlinearity is not a necessity for invariant propagation; indeed, he proved that Bessel beams can propagate in a purely linear material without deformation (Durnin, 1987; Dallaire et al., 2009). On the other hand, Lopez and Helmerson have recently demonstrated that non-diffracting beams with arbitrary transverse shapes can be generated (Lopez-Mariscal and Helmerson, 2010). They have tailored a nondiffracting beam of an arbitrary pattern via an azimuthal modulation of the angular spectra of Helmholtz-Gauss wave fields.

The diffraction-free character of ideal Bessel beams has triggered immense interest, though, it was soon realized that, due to practical considerations, only quasiBessel beams can be produced (Durnin, 1987; Durnin et al., 1987). Gori et al. derived the complete solution describing the evolution of transverse Bessel-Gauss beams during propagation (Gori et al., 1987). Bessel and Bessel-Gauss beams have been widely discussed in the literature because of their potential applications in optical alignment, surveying, and optical interconnections (Abramochkin and Volostnikov, 1993; Cha'vez-Cerda et al., 1996; Paterson and Smith, 1996; Piestun and Shamir, 1998; Gutie'rrez-Vega et al., 2000; Gutiérrez-Vega et al., 2003; Tsangaris et al., 2003).

There are several passive and active methods for production of Bessel-like beams. For instance, passive optical systems fed by laser light using a ring aperture and a positive lens (Durnin, 1987; Durnin et al., 1987), refractive or diffractive axicons (Indebetouw, 1989; Scott and McArdle, 1992), holographic methods (Turunen et al., 1988; Vasara et al., 1989), Fabry-Perot interferometers (Horváth et al., 1997), or diffractive phase elements (Cong et al., 1998). Active schemes to produce Bessel-type modes in laser resonators include, e.g., arrangements based on annular intracavity elements (Durnin and Eberly, 1989; Litvin and Forbes, 2008),

*Corresponding author. E-mail: s_batebi@guilan.ac.ir.

PACS: 42.60.By; 42.60.Da; 42.55.Xi; 42.55.Rz; 42.60.Jf. 


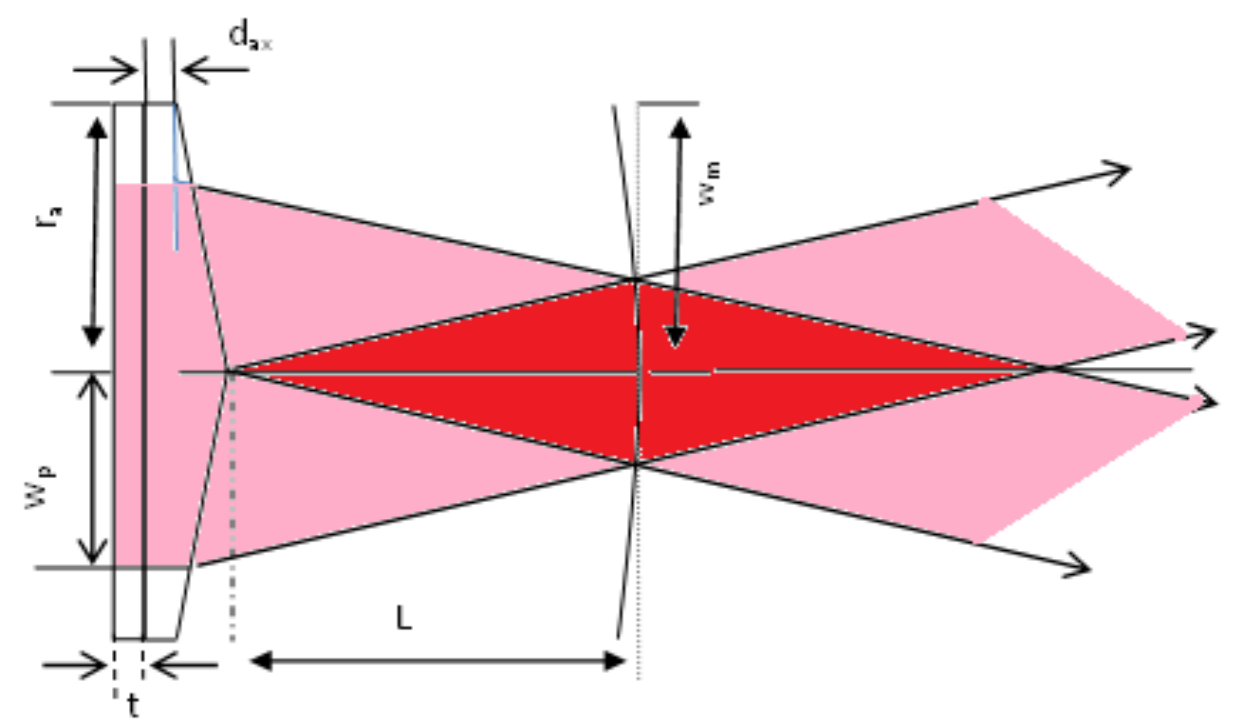

Figure 1. The configurations of solid state thin disk laser with an axicon-based resonator.

output mirror with annular apertures to produce conical fields (Uehara and Kikuchi, 1989), graded-phase mirrors (Pääkkönen and Turunen, 1998), and diode pumped $\mathrm{Nd}$ :YAG lasers (Hakola et al., 2004). Axicon-based resonators supporting Bessel or Bessel-Gauss beams were proposed independently by Rogel-Salazar et al. (2001) and Khilo et al. (2001). Their studies continued and extended by Gutiérrez-Vega et al. (2003), Tsangaris et al. (2003) and Hernández-Aranda et al. (2005).

On the other hand, power scalability and minimal thermal lensing are the particular features of the thin-disk laser (TDL). High output power, high efficiency and good beam quality are further advantageous qualities of this type of solid-state lasers (Giesen et al., 1994). The highest reported output power of a Yb:YAG single disk is $5.3 \mathrm{~kW}$ in multi-mode operation (Giesen and Speiser, 2007).

In our previous work, we considered a passive axiconbased configuration to generate Bessel-like beam with a flat output mirror (Aghbolaghi et al., 2010). In this work, an axicon based disk type laser with spherical output mirror is numerically studied. This numerical model is performed in two steps; at first, the transverse profile of beam is obtained employing the Collins integral and at the second step, the photon rate equation accounts for the amplification and saturation effect. These two steps are repeated successively approaching a steady state. We calculated the output power, intensity and phase distribution on rear surface of the axicon and output coupler.

\section{CONFIGURATION}

The configuration of a solid state thin disk laser with a
Bessel-Gauss resonator is shown in Figure 1. It consists of a composite Yb:YAG/YAG or Yb:YAG/Diamond crystal and a concave spherical output mirror separated by distance $\mathrm{L}$. The composite crystal includes a Yb-doped thin disk as the active medium and a conical-shaped undoped part (pure YAG) as an axicon with an apex angle $Y$. Such as the composite laser martials (Yb:YAG/YAG), this structure can be made through heat diffusion bonding technique (Dashkasan et al., 2012). The back surface of the active medium is coated with a high reflection layer which acts as a back mirror of resonator. $r_{a}, t, d_{a x}$, and $\mathrm{R}$ denote, respectively, the radius of the axicon, thickness of the active medium, thickness of the axicon, and curvature radius of the output coupler. We assume the refractive indices of the active medium and the axicon are the same. Also, we suppose $d=t+$ $\mathrm{d}_{\mathrm{ax}}$.

It is customary to replace each end mirror by two lenses with focal length $f=R$ and locate the reference planes between them. The ABCD ray transverse matrix between two reference planes is obtained as follows:

$\mathrm{M}_{\mathrm{ao}}=\left[\begin{array}{cc}\mathrm{g}_{1}^{*} & \mathrm{~L}^{*} \\ \frac{\mathrm{g}_{1}^{*} \mathrm{~g}_{2}^{*}-\mathrm{n}}{\mathrm{L}^{*}} & \mathrm{~g}_{2}^{*}\end{array}\right]$

where

$\mathrm{g}_{1}^{*}=1-\frac{(\mathrm{n}-1) \mathrm{Y}}{\mathrm{r}} \mathrm{L}$

$g_{2}^{*}=-\frac{1}{R}\left(d+\left(r_{a}-r\right) Y\right)+\left(1-\frac{1 L}{R}\right)\left(-\frac{(n-1) Y}{r}(d+\right.$

$\left.\left.\left(r_{a}-r\right) y\right)+n\right)$

$L^{*}=d+\left(r_{a}-r\right) Y+\left(-\frac{(n-1) Y}{r}\left(d+\left(r_{a}-r\right) Y\right)+n\right) L$

where, $r$ is the distance of the ray from the optical axis 


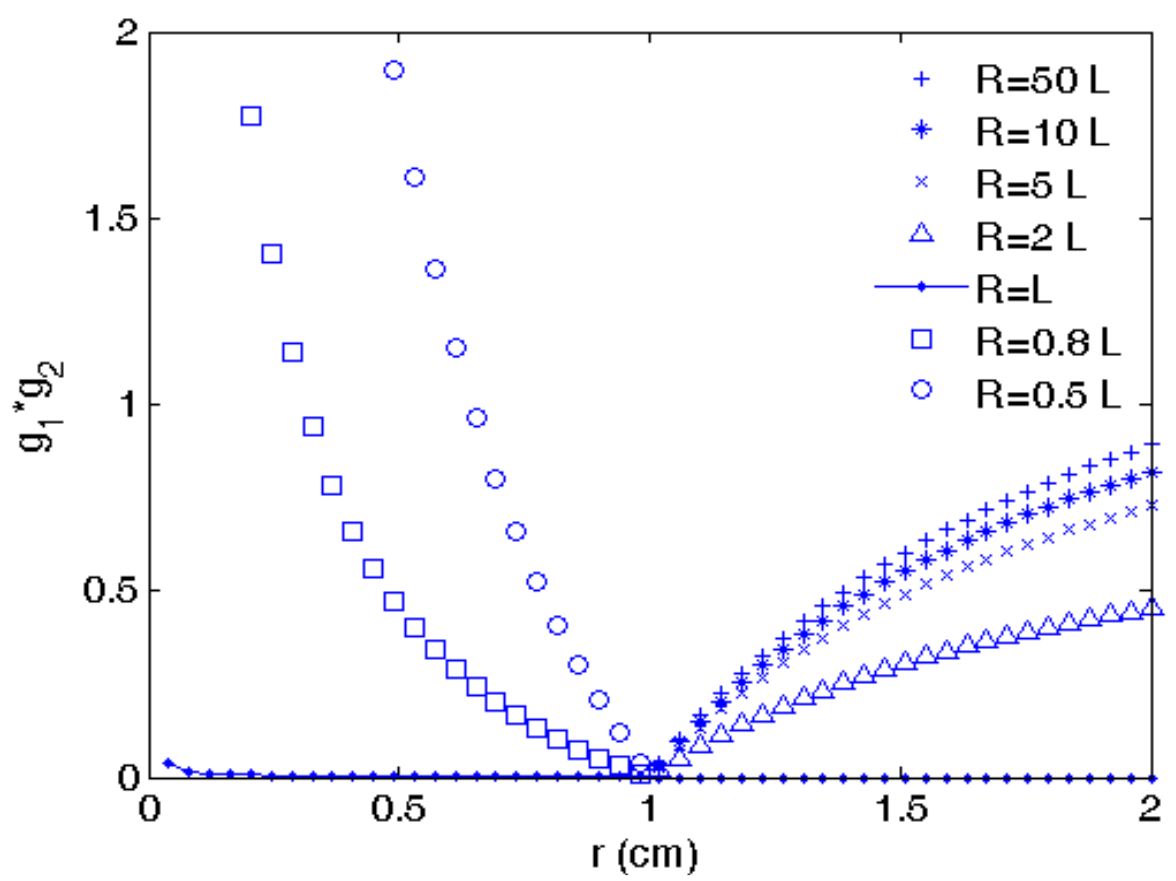

Figure 2. The stability of an axicon-based resonator versus position of ray on the axicon for different radii of the curvature of the output coupler.

and $\mathrm{n}$ is the refractive index of the axicon and disk. Stability condition is given by:

$-1<\frac{1}{n}\left(2 g_{1}^{*} g_{2}^{*}-n\right)<1$ or $0<g_{1}^{*} g_{2}^{*}<n$

The stability parameter of this resonator versus the radius of the axicon for different curvature radius is shown in Figure 2. It should be noted that the following parameters are used in our calculations:

$\mathrm{Y}=0.5^{\circ}=8.7 \mathrm{mrad}$

$\mathrm{n}=1.82(\mathrm{Yb}: \mathrm{YAG})$

$r_{a}=20 \mathrm{~mm}$

The cavity length $L=782.4 \mathrm{~mm}$ is calculated according to the resonance condition $\left(L=r_{a} / 2(n-1) \gamma\right)$. The point $r=\frac{r_{a}}{2}=1 \mathrm{~cm}$ that locates in the intersection of curves corresponds to the eigenvalue driven by Gutie'rrez-Vega (2003).

\section{Diffraction}

The Huygens-Fresnel diffraction integral may be expressed in terms of the ABCD matrix elements (Collins, 1970). The incident amplitude distribution $u_{l p}\left(\rho_{n}, \theta_{n}\right)$, and the diffracted one $u_{l p}\left(\rho_{m}, \theta_{m}\right)$ on two reference planes separated by a distance $L$ are interconnected by the following integral:

$$
\begin{aligned}
& u_{l p}\left(\rho_{n}, \theta_{n}\right)=\frac{-i k}{2 \pi B} \exp (-i k L) \\
& \times \iint u_{l p}\left(\rho_{m}, \theta_{m}\right) \exp \left\{\frac { - i k } { 2 \pi B } \left[\mathrm{A} \rho_{m}^{2}+\mathrm{D} \rho_{n}^{2}-2 \rho_{m} \rho_{n} \cos \left(\theta_{m}-\right.\right.\right. \\
& \left.\left.\left.\theta_{n}\right)\right]\right\} \rho_{m} d \rho_{m} d \theta_{m}
\end{aligned}
$$

where $\rho_{m}$ and $\theta_{m}$ are the cylindrical coordinates of the incident plane; $u_{l p}\left(\rho_{m}, \theta_{m}\right)=u_{l p}\left(\rho_{m}\right)\left\{\begin{array}{l}\cos l \theta_{m} \\ \sin l \theta_{m}\end{array}\right.$ is the incident optical field; $u_{l p}\left(\rho_{n}, \theta_{n}\right)$ is the diffracted optical field, $\lambda$ is the light wavelength, $k$ is the wavenumber given by $k=2 \pi / \lambda$, and $A, B$, and $D$ are elements of the ray matrix. In addition, an angular dependency is introduced making it possible to simulate transverse-mode diffraction. Integration over $\theta_{\mathrm{m}}$, employing $\int_{0}^{2 \pi} \exp (i \alpha \cos \theta) \cos 1 \theta d \theta=$ $2 \pi \mathrm{i}^{\mathrm{l}} \mathrm{J}_{1}(\alpha), \int_{0}^{2 \pi} \exp (i \alpha \cos \theta) \sin l \theta d \theta=0$ results in:

$$
\begin{aligned}
& u_{l p}\left(\rho_{n}, \theta_{n}\right)=\int_{\rho_{1}=0}^{w_{m}} K_{l}\left(\rho_{m}, \rho_{n}, \theta_{n}\right) T_{m}\left(\rho_{m}\right) u_{l p}\left(\rho_{m}\right) d \rho_{m} \\
& K_{l}\left(\rho_{m}, \rho_{n}, \theta_{n}\right)= \\
& i^{l+1}\left(\frac{k}{B}\right) \rho_{m} \exp (-i k(L+ \\
& \left.\frac{D}{2 B} \rho_{n}^{2}\right)\left\{\begin{array}{l}
\cos l \theta_{2} \\
\sin l \theta_{2}
\end{array} \exp \left(\frac{-i k A}{2 B} \rho_{\mathrm{n}}^{2}\right) J_{l}\left(\frac{k}{B} \rho_{n} \rho_{m}\right) .\right.
\end{aligned}
$$

The upper limit $w_{m}$ corresponds to the radius of the aperture of the first reference plane. A transform factor $T_{m}\left(\rho_{m}\right)$ is called the transmittance function of the optical element or aperture and $J_{l}(x)$ is the $l$ th-order 
Bessel function. This makes it possible to reduce the twodimensional integral to only one dimension. Finally, time dependency can be expressed by the factor of exp $(-i \omega t)$. The constant phase shift of exp $(-i k L)$ can be ignored in the integral equation (Equation 5).

\section{AMPLIFICATION}

First, we assume that the amplifier is made from a thin disk within which we neglect diffraction. The level scheme in Yb: YAG can be considered as quasi 4 level scheme. But the level system can be treated as two level system under the assumption that there are fast relaxations between the levels within one manifold compared to the fluorescence lifetime of the laser transition (Ostermeyer and Straesser, 2007; Bordet and Bartniki, 2006). The rate equations for the pump process can be formulated for the population density of the upper and the lower manifolds with the emission and absorption cross sections for the pump at $941 \mathrm{~nm}$ and the laser at $1030 \mathrm{~nm}$. Here, we define some parameters and recall the formula obtained in (Bordet, 2000; Bordet and Bartniki, 2006). The back reflection regenerative pumping is used in this work. The absorbed pump intensity can be written as:

$$
\begin{aligned}
& \mathrm{I}_{\mathrm{abs}}(0)=\mathrm{I}_{\mathrm{p}}^{+}(0) \mathrm{B}(\Gamma) \\
& \mathrm{B}(\Gamma)=\frac{(1-\Gamma)\left(1+\mathrm{R}_{\mathrm{m}}^{\mathrm{p}} \Gamma\right)}{1-\gamma \mathrm{R}_{\mathrm{m}}^{\mathrm{p}} \Gamma^{2}}
\end{aligned}
$$

where $\gamma$ is the transmission of the recycling apparatus taking into account the reflection-transmission of the optical components of the recycling loop and the mode matching of pump beam, $\mathrm{R}_{\mathrm{m}}^{\mathrm{p}}$ is back reflection of the active medium at pump wavelength, and the saturated single pass transmission of the amplifier medium, $\Gamma$, is given by

$\Gamma=G^{\alpha_{0} / g_{0}} \exp \left(-\alpha_{0}\left(f_{p}-f_{l}\right) d\right)$

where $G$ and $d$ are the gain saturated and thickness of the active medium, respectively. Also, $f_{p}, f_{l}, \alpha_{0}$ and are $g_{0}$ given by the following equations:

$f_{p}=\frac{f_{l 1}}{f_{l 1}+f_{u j}}$

$f_{l}=\frac{f_{l m}}{f_{l m}+f_{u 1}}$

$g_{0}=\sigma_{l} N_{Y b}\left(f_{l k}+f_{u 1}\right)$,

$\alpha_{0}=\sigma_{p} N_{Y b}\left(f_{l 1}+f_{u j}\right)$

$\sigma_{p}$ and $\sigma_{l}$ are respectively the absorption and emission cross-sections, $N_{Y b}$ is the ytterbium concentration, $f_{l i}$ and $f_{u j}$ are the Boltzmann occupation factors of the Stark levels $i$ and $j$ of the lower and upper levels, respectively. Let us call $I_{i}$ impinging laser intensity with medium. The amplification of the intensity of the beam reads as $I_{i+1}=I_{i} G^{2}$. After the beam is amplified, the saturated gain can be computed by (Ostermeyer and Straesser, 2007):

$$
(G-1)\left(1+R_{m}^{l} G\right) I_{i+1}(d)+\ln G-g_{0}\left\{\frac{I_{p}^{+}(0) B(\Gamma)}{\alpha_{0}}-f_{l} d\right\}=0
$$

where $\mathrm{R}_{\mathrm{m}}^{\mathrm{l}}$ and $\mathrm{d}$ are the reflectivity of back side of active medium and thickness of it, respectively. Note that $I_{p}$ and $I_{l}$ are normalized to the corresponding saturation intensities.

\section{NUMERICAL CALCULATIONS}

The calculation process is carried out as follows: At the first reference plane, that is, on the boundary surface between the active medium and the axicon, random distributions for both the amplitude and the phase are chosen. This light is transmitted through the axicon and then is propagated in free space toward the output coupler then reflected and propagated backward to the axicon. Finally, we calculated the double-pass amplification through the active medium. This procedure is successively repeated until a steady state is obtained. In our analysis, we ignored deformation and thermal lensing of the active medium.

\section{Phase and intensity distribution}

The loss of fundamental mode on the geometrical parameters of the cavity was examined with the quantities given in Table 1. For the radius of the pump area, $r_{p}=7 \mathrm{~mm}$, the resonance condition gives the cavity length as $\mathrm{L}=49.19 \mathrm{~cm}$.

For our configuration, typically, several hundred round trips are required in order for iteration to converge starting from an arbitrary rotational symmetric field distribution on refractive axicon. The intensity distribution and phase of the field at the surface of the active medium (just before the axicon) are shown in Figure $3 a$ and $b$ for flat output mirror, and in Figure $4 a$ and $b$, for a concave spherical mirror with $R=50 \mathrm{~L}$. They cover a broad area of the active medium. The power density distributions are shown versus the radius of the pump area $r$ in deferent curvature of the output coupler in Figure $5 a$ and $b$ for $R>$ $\mathrm{L}$ and $\mathrm{R}<\mathrm{L}$, respectively. It can be seen that for curvature radii $R$ smaller than resonator length $L$ the power density distributions are strongly narrow. Specifically for $R=L$, it is distributed narrowly around $r / 2$. On the other hand, for $R>L$, the intensity distributions spread over large areas. Thus, in order to use the maximum gain area, it is most reasonable to choose output couplers with the radius curvatures greater than the length of cavity. It can be very important for power scaling. As expected, the phase curve corresponds 
Table 1. Parameters of the disk laser with axicon based resonator.

\begin{tabular}{lcc}
\hline Parameter & Symbol & Value \\
\hline Axicon angle (degree) & $\mathrm{V}$ & $0.5^{\circ}$ \\
Radius of disk (cm) & $\mathrm{r}_{\mathrm{a}}$ & 1 \\
Thickness of disk $(\mathrm{cm})$ & $\mathrm{t}$ & 0.02 \\
Radius of pump area & $\mathrm{r}_{\mathrm{p}}$ & 0.7 \\
Dopant at. & $\mathrm{N}$ & $10 \%$ \\
Pump power $(\mathrm{w})$ & $\mathrm{P}_{\mathrm{pump}}$ & 1800 \\
Pump wavelength $(\mathrm{nm})$ & $\lambda_{\mathrm{p}}$ & 941 \\
Laser wavelength $(\mathrm{nm})$ & $\lambda_{\mathrm{l}}$ & 1030 \\
Refractive index of axicon & $\mathrm{n}_{\mathrm{ax}}$ & 1.8153 \\
Refractive index of active medium & $\mathrm{n}_{\mathrm{ac}}$ & 1.8154 \\
Absorption cross sections at pump wavelength $\left(\mathrm{cm}^{2}\right)$ & $\sigma_{\mathrm{p}}$ & $0.719 \times 10^{-20}$ \\
Emission cross sections at laser wavelength $\left(\mathrm{cm}^{2}\right)$ & $\sigma_{\mathrm{l}}$ & $2.2 \times 10^{-20}$ \\
The population of the lowest sublevel of the fundamental manifold & $\mathrm{f}_{\mathrm{l}}$ & 0.88 \\
The population of the sublevel $\mathrm{j}$ of the excited state & $\mathrm{f}_{\mathrm{uj}}$ & 0.17 \\
The population of the terminal laser transition level & $\mathrm{f}_{\mathrm{lk}}$ & 0.046 \\
The population of the lowest level of the upper manifold & $\mathrm{f}_{\mathrm{u} 1}$ & 0.672 \\
Yb ions doping $(\%)$ & $\mathrm{c}_{\mathrm{yb}}$ & 10 at $\%$ \\
Lifetime $(\mu \mathrm{s})$ & $\mathrm{T}_{\mathrm{u}}$ & 950 \\
\hline
\end{tabular}

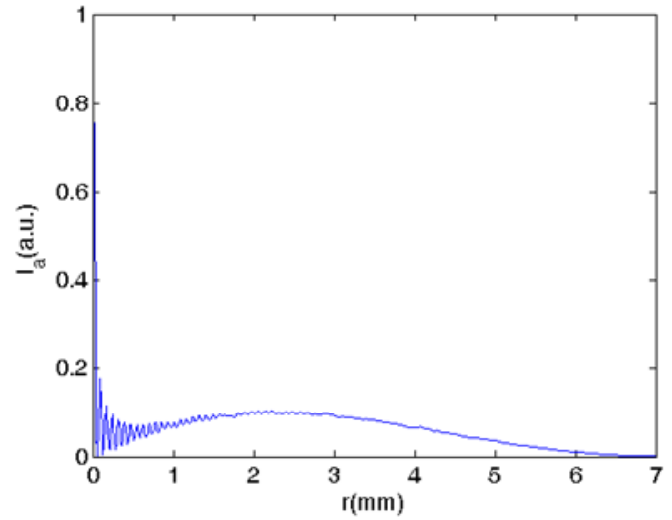

(a)

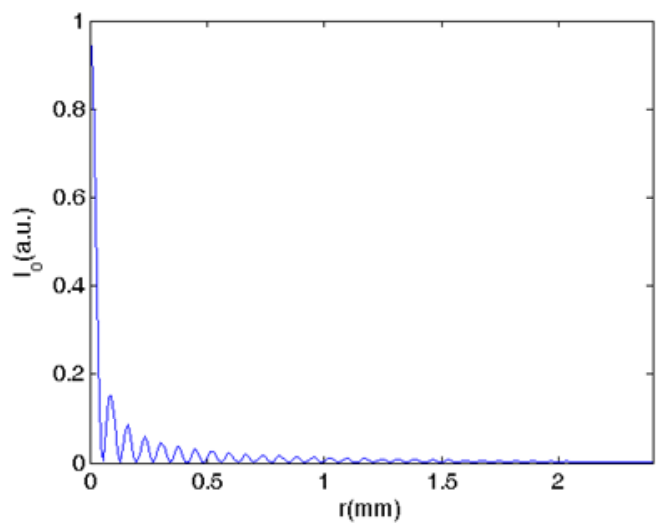

(c)

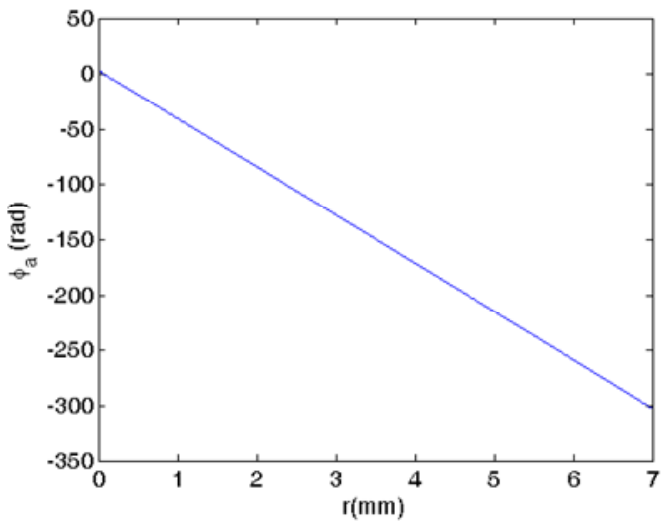

(b)

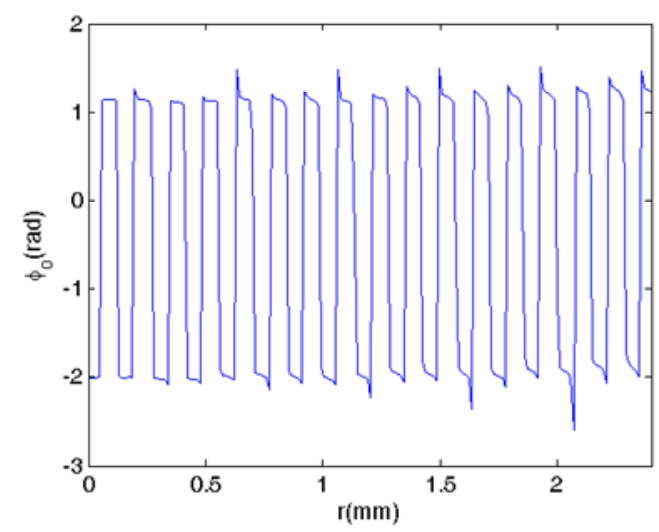

(d)

Figure 3. The intensities transverse distribution (a) and optical phase distribution (b) on the active medium, the intensity transverse distribution (c) and optical phase distribution (d) on the output coupler. 


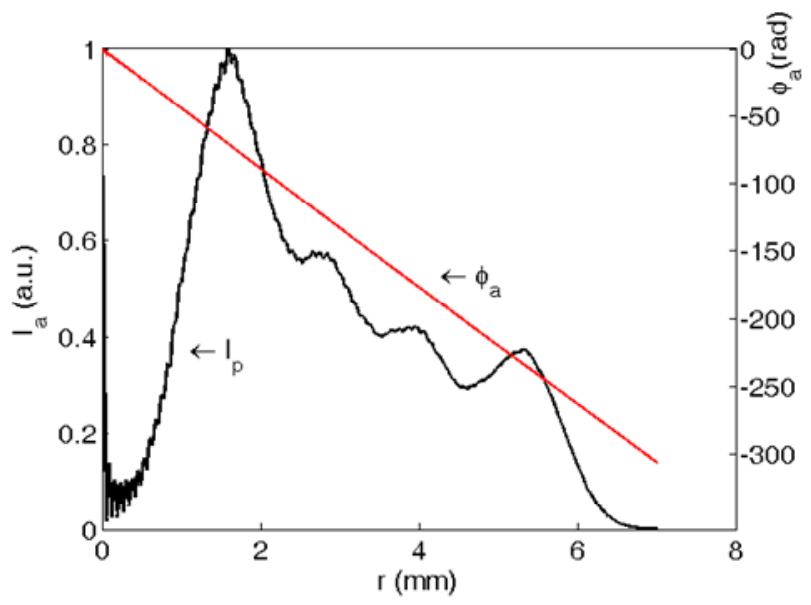

(a)

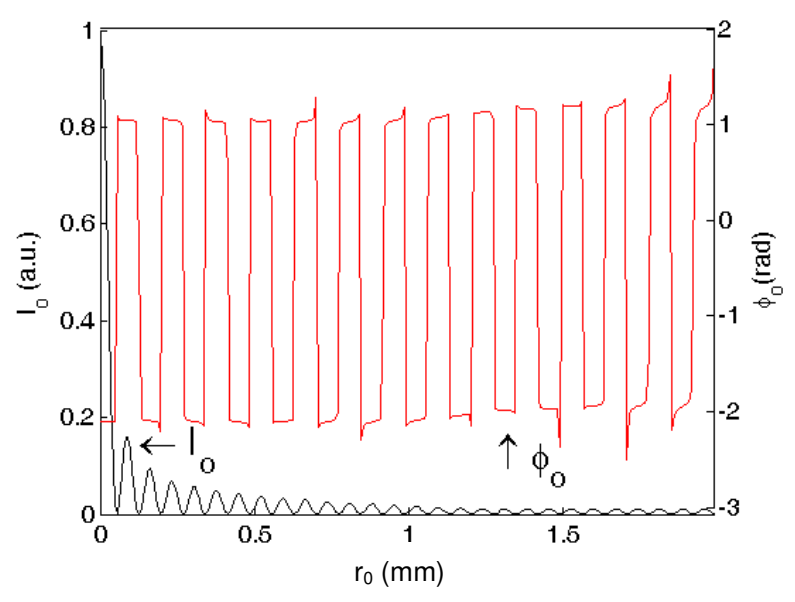

(b)

Figure 4. The intensities transverse distribution on the active medium and output coupler (a), the intensity transverse distribution and its phase on output coupler (b).

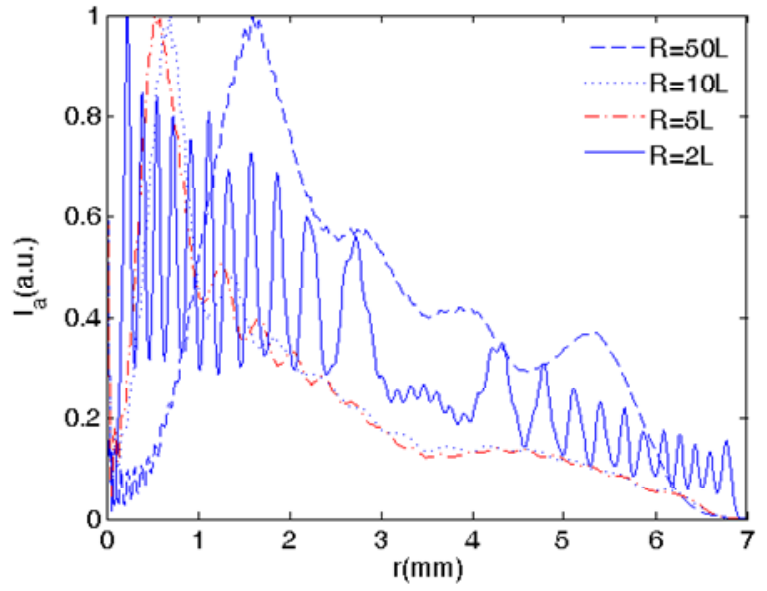

(a)

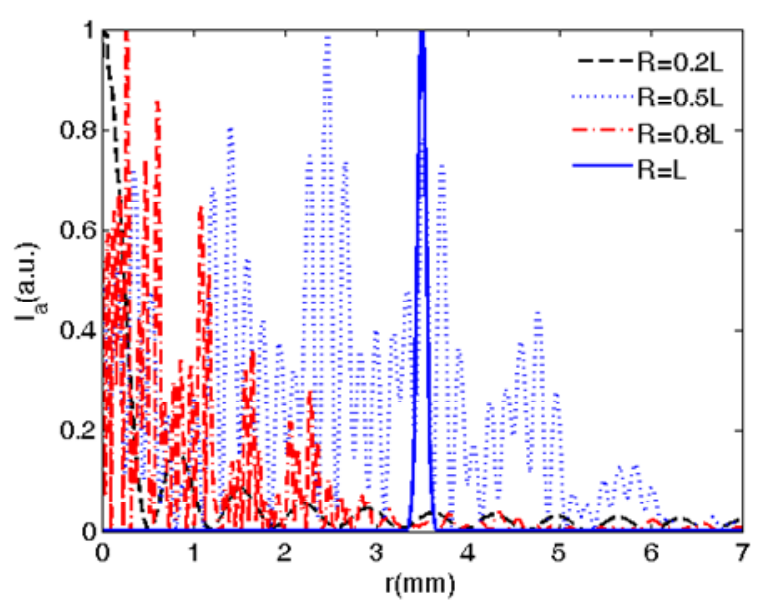

(b)

Figure 5. Schematic representation of the intensities transverse distribution on the active medium in term of radius.

almost exactly to the linear equation $\theta(r)=-k(n-1) \gamma r$ for both flat and curved output couplers; this fact confirms that the field at the axicon behaves as a conic wave.

The output field at flat output mirror (Figure $3 \mathrm{c}$ and $\mathrm{d}$ ) corresponds to the calculated mode. It can be observed that the lowest mode in resonator falls off faster than the ideal zero-order Bessel beam $\mathrm{J}_{0}\left(\mathrm{k}_{\mathrm{t}} \rho\right)$. It becomes virtually zero outside the interval $\left[0, r_{p} / 2\right]$. In Figure $3 d$, the transverse phase distribution is represented. Similar to an ideal Bessel beam, the phase pattern jumps of $\pi$ at the zeros of the Bessel function. The output field and its phase in this configuration with spherical mirror are shown in Figure 4b. The field has a Bessel shape, but now the Gaussian modulation is more evident.

\section{Effects of varying the geometrical parameters on the loss}

The loss per round trips with respect to the transverse radius of output mirror $w_{m}$, axicon radius $r_{a}$ or $w_{p}$ and cavity length is investigated. In Figure $6 a$, the results of wave optics show that the loss decreases drastically versus $\mathrm{w}_{\mathrm{m}}$ and approaches zero at $\mathrm{w}_{\mathrm{m}}=\mathrm{w}_{\mathrm{p}}$. We used it in this work. According to Figure $6 \mathrm{~b}$, it slowly varies versus $w_{p}$ at large amount and change fast at small $w_{p}$. It can be useful for the disk laser configuration with a single active medium. The loss in terms of the cavity length and the wedge angle of the axicon are represented in Figure $6 \mathrm{c}$ and $\mathrm{d}$. The minimum loss happens at the resonance 


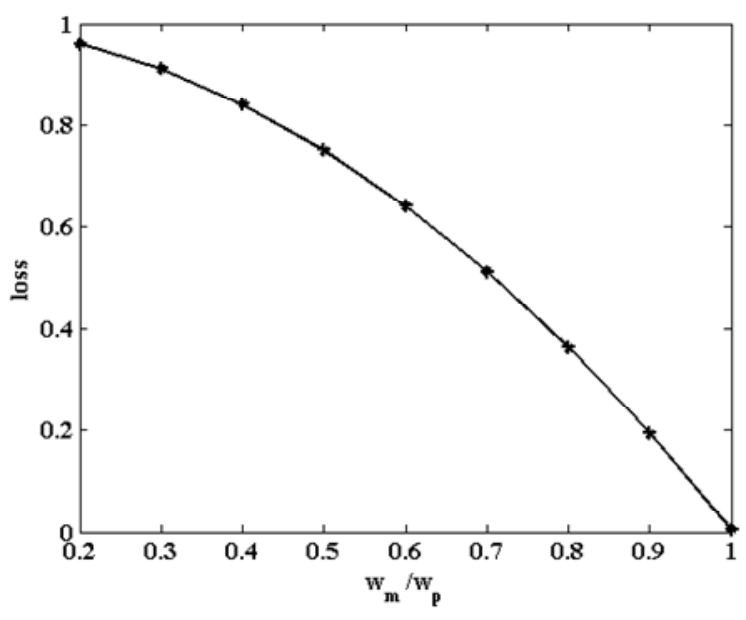

(a)

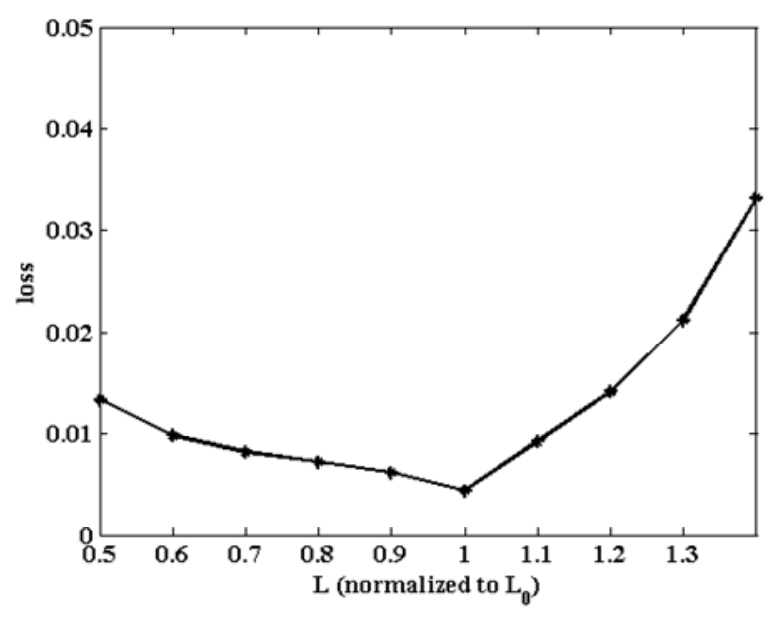

(c)

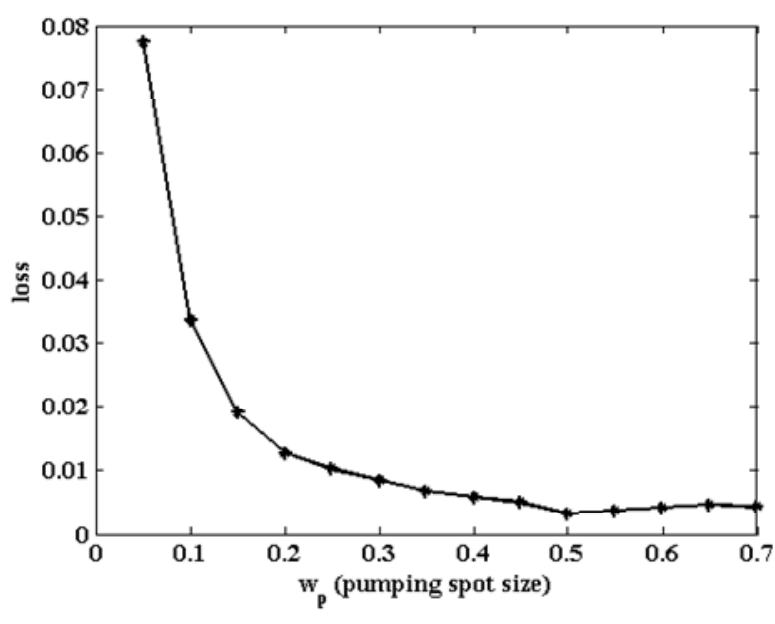

(b)

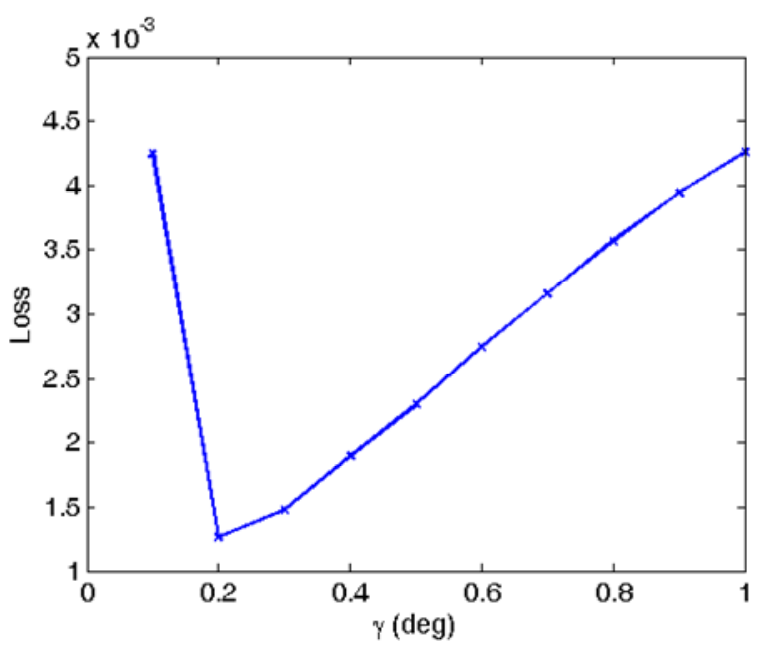

(d)

Figure 6. The loss versus $w_{\mathrm{m}}$. It has been normalized to the pumping spot size (a), the loss versus pumping spot size (b), the loss in terms of the cavity length (c), the loss in terms of the wedge angle of the axicon (d).

condition (distance $L_{0}$ ). The rate of loss increases at $L>$ $L_{0}$ is faster than it at $L<L_{0}$, as we expect, because the cavity length decreases.

Let us consider the effect of varying the curvature radius of the output mirror. Consider the loss behavior corresponding to the lowest mode resonating within the cavity. The loss as a function of the normalized R/L is depicted in Figure 7. The dashed line represents the loss in the limit by left and bottom axis when $\mathrm{R}$ is greater than $\mathrm{L}$. The solid line represents it by right and above axis when $R$ is smaller than $L$. In spite of Ref.13, the resonator behavior in range $R \in[\mathrm{L}, 10 \mathrm{~L}]$ is investigated, where the loss of resonator fluctuated and has some extremums. It may be related to shift frequency in it because of varying the radius curvature of the output coupler. The loss is minimum about $\mathrm{R}=\mathrm{L}$. In range, $\operatorname{R\in }(0, \mathrm{~L}]$, based on our simulation the output beam profile is nearly far from the Bessel-Gauss beam. Our numerical results have good agreement with Ref. 13 in large $R$.

\section{Output power}

We employed the configuration of Figure 1 as a thin disk with axicon Bessel-Gauss resonator. It is assumed that $10 \% \mathrm{Yb}$ doped in YAG crystal as an active medium. We calculated absorption (for the back reflection regenerative pumping), the diffraction and amplification into cavity by equations mentioned earlier. The required information for calculating is given in Table 1. The transverse intensity profile on the active medium (black curve) on left and bottom axis and the phase of field (red curve) on right 
$\mathrm{R} / \mathrm{L}$

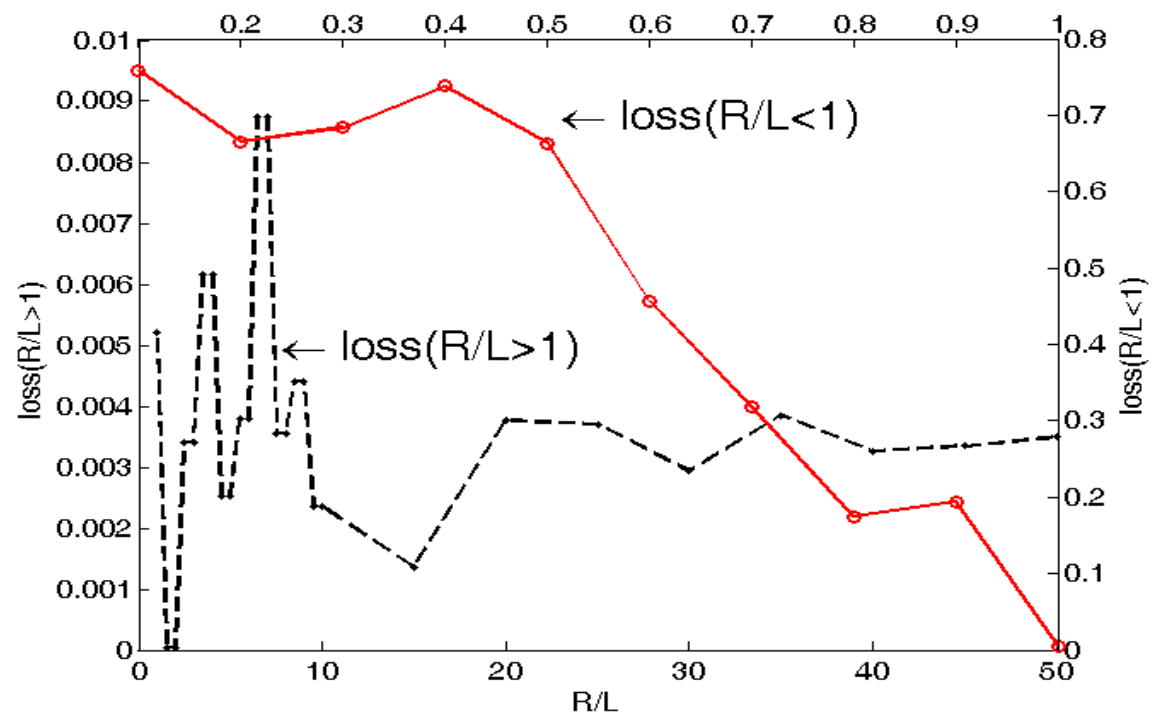

Figure 7. The dashed line represents the loss in the limit by left and bottom axis when $R / L$ is greater than one. The solid line represents it by right and above axis when $R / L$ is smaller than one.

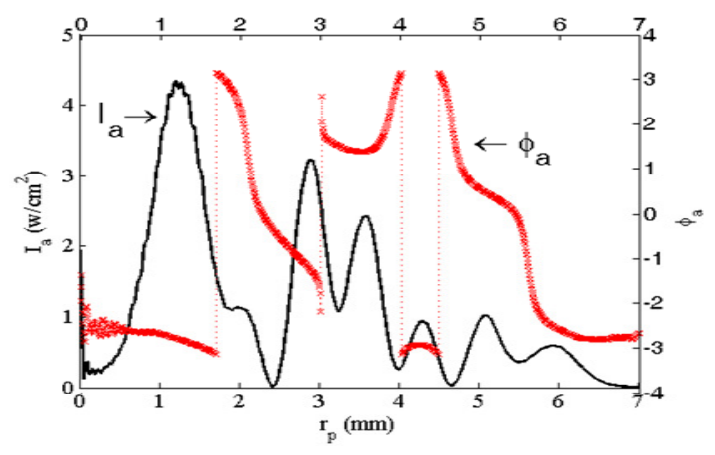

(a)

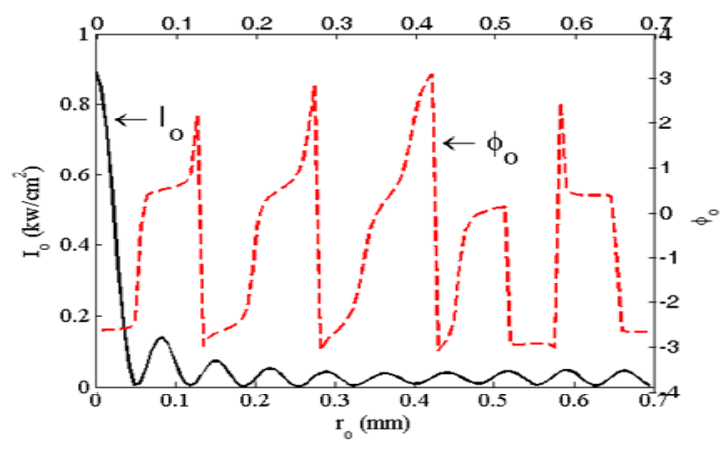

(b)

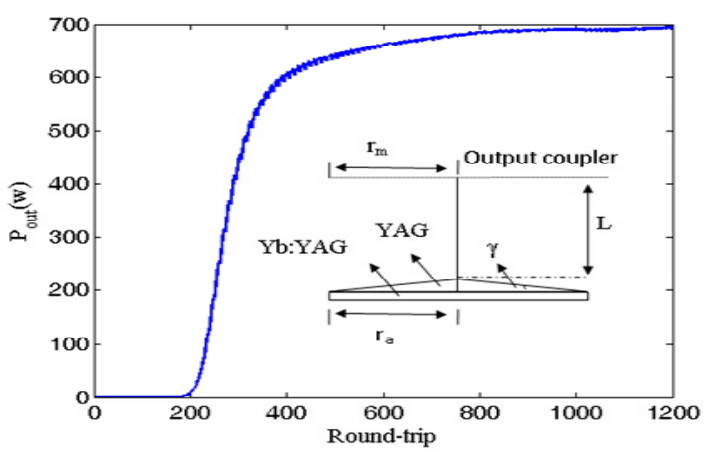

(c)

Figure 8. The transverse intensity distribution $\mathrm{l}_{a}$ and phase $\varnothing_{\alpha}$ on the active medium, (a), The transverse intensity distribution $I_{0}$ and $\emptyset_{0}$ on the output coupler, (b), and output power in term of number of roun-trips, (c).

and top axis are shown in Figure 8a. The output power density (black curve) is a high power and intense BG beam that is shown on the output coupler on left and bottom axis. The phase of field is shown by red curve on 
right and top axis in Figure 8b.

\section{CONCLUSION}

We have presented the thin disk solid state laser with Bessel and BG resonator. We have described analytically stable and unstable area by geometrical optics viewpoint in term of different geometrical parameters. The traditional Gaussian beam resonator in which the spot size of the fundamental mode on the active media is very small. on the other hand, the spot size on the active medium in this configuration is very large. It can be very important for the disk laser configuration with a single active medium. The loss of resonator per round trips is calculated in terms of different resonator parameters by the viewpoint of wave optics. These results illustrated this configuration can be used for thin disk lasers that their gain per round trip is low. In this configuration, based on our computer model, a high power BG laser beam with small spot size can be obtained.

\section{ACKNOWLEDGEMENTS}

The collaboration of Sazegari V, Shayeganmanesh $M$, Mollai $\mathrm{J}$ and Jafari $\mathrm{A}$, are gratefully acknowledged.

\section{REFERENCES}

Abramochkin E, Volostnikov V (1993). Spiral-type beams. Opt. Commun. 102:336-350.

Aghbolaghi R, Mollai J, Batebi S, KhayatJafari A (2010). Numerical study of axicon-based Bessel-Gauss resonator for thin disk laser, Proc. SPIE Vol. $774777471 \mathrm{H}-1$.

Bordet GL (2000). Theoretical investigation of quasi-three-level longitudinally pumped continuous wave lasers. Appl. Opt. 39:966971.

Bordet GL, Bartniki E (2006). Generalized formula for continuous-wave end-pumped $\mathrm{Yb}$-doped material amplifier gain and laser output power in various pumping configurations. Appl. Opt. 36:9203-9209.

Cha'vez-Cerda S, McDonald GS, New GHC (1996). Nondiffracting beams: travelling, standing, rotating and spiral waves. Opt. Commun. 123:225-233.

Collins Jr. SA (1970). Lens-system diffraction integral written in term of matrix optics. J. Opt. Soc. Am. A 60:1168-1177.

Cong WX, Chen NX, Gu BY (1998). Generation of nondiffracting beams by diffractive phase elements. J. Opt. Soc. Am. A 15:2362-2364.

Dallaire M, McCarthy N, Piche M (2009). Spatiotemporal Bessel beams: Theory Exp. 17:18148-18158.

Dashkasan MJ, Barati E, Aghbolaghi R (2012). Designing of and efficient multi-appertureadge-pumped thin disk laser. Opt. Laser Technol. 44:800-805.

Durnin AJ (1987). Exact solutions for nondiffracting beams. I. The scalar theory. J. Opt. Soc. Am. A 4:651-654.

Durnin J, Eberly JH (1989). Diffraction free arrangement. U.S. patent 4:885-887.

Durnin J, Micely Jr. JJ, Eberly JH (1987). Diffraction free beams. Phys. Rev. Lett. 58:1499-1501.
Giesen A, Hugel H, Wittig K, Brauch U, Opower H (1994). Scalable concept for diode-pumped high-power solid-state lasers. Appl. Phys. B 58:365.

Giesen A, Speiser J (2007). Fifteen years of work on thin-disk lasers: results and scaling laws. IEEE JSTQE 13(3):598-609.

Gori F, Guattari G, Padovani C (1987). Bessel-Gauss beams. Opt. Commun. 64:491-495.

Gutie'rrez-Vega JC, Iturbe-Castillo MD, Chavez-Cerda S (2000). Alternative formulation for invariant optical fields: Mathieu beams. Opt. Lett. 25:1493-1495.

Gutiérrez-Vega JC, Rodríguez-Masegosa R, Chávez-Cerda S (2003). Bessel-Gauss resonator with spherical output mirror: geometrical and wave-optics analysis. J. Opt. Soc. Am. A 20:2113-2122.

Hakola A, Buchter SC, Kajava T, Elfström H, Simonen J, Pääkkönen P, Turunen J (2004). Bessel-Gauss output beam from a diode-pumped NdYAG laser. Opt. Commun. 238:335-340.

Hasegawa A, Tapert F (1973). Transmission of stationary nonlinear optical pulse in dispersive dielectric fibers: I. Anomalous dispersion. Appl. Phys. Lett. 23:142-144.

Hernández- Aranda RI, Chávez -Cerda S, Gutiérrez-Vega JC (2005). Theory of the unstable Bessel resonator, 22:1909-1916.

Horváth ZL, Erdélyi M, Szabó G, Bor ZS, Tittel FK, Cavallaro JR (1997). Generation of nearly nondiffracting Bessel beams with a Fabry-Perot interferometer. J. Opt. Soc. Am. A 14:3009-3013.

Indebetouw G (1989). Nondiffracting optical fields: some remarks on their analysis and synthesis. J. Opt. Soc. Am. A 6:150-152.

Khilo AN, Katranji EG, Ryzhevich AA (2001). Axiconbased Bessel resonator: analytical description and experiment. J. Opt. Soc. Am. A 18:1986-1992.

Litvin IA, Forbes A (2008). Bessel- Gauss resonator with internal amplitude filter. Opt. Commun. 281:2385-2392.

Lopez-Mariscal C, Helmerson K (2010). Shaped nondiffracting beams. Opt. Lett. 35:1215-1217.

Mollenaure LF, Stolen RH, Gordon JP (1980). Experimental observation of picoseconds pulse narrowing and solitons in optical fiber. Phys. Rev. Lett. 45:1095-1098.

Ostermeyer M, Straesser A (2007). Theoretical investigation of feasibility of $\mathrm{Yb}$ : YAG as laser material for nano second pulse emission with large energies in the Joule range. Opt. Commun. 274:422-428.

Pääkkönen P, Turunen J (1998). Resonators with Bessel-Gauss modes. Opt. Commun. 156:359-366.

Paterson C, Smith R (1996). Helicon waves: propagation invariant waves in a rotating coordinate system. Opt. Commun. 124:131-140.

Piestun R, Shamir J (1998). Generalized propagation invariant wave fields. J. Opt. Soc. Am. A 15:3039-3044.

Rogel-Salazar J, New GHC, Chávez-Cerda S (2001). Bessel-Gauss beam optical resonator. Opt. Commun. 190:117-122.

Scott G, McArdle N (1992). Efficient generation of nearly diffraction-free beams using an axicon. Opt. Eng. Bellingham 31:2640-2643.

Tsangaris CL, New GHC, Rogel-Salazar J (2003). Unstable Bessel beam resonator. Opt. Commun. 223:233-238.

Turunen J, Vasara A, Friberg AT (1988). Holographic generation of diffraction-free beams. Appl. Opt. 27:3959-3962.

Uehara K, Kikuchi H (1989). Generation of nearly diffraction-free laser beams. Appl. Phys. B 48:125-129.

Vasara A, Turunen J, Friberg AT (1989). Realization of general nondiffracting beams with computer-generated holograms. J. Opt. Soc. Am. A 6:1748-1754. 